\title{
REMARKS ON A CERTAIN HYPOTHESIS IN SINGULAR PERTURBATIONS
}

\author{
K. W. $\mathrm{CHANG}^{1}$
}

Our aims are to prove the following theorem and to apply the result to remove a hypothesis (hypothesis (A) below) which has been imposed in many papers on singular perturbations.

Theorem. Let $\epsilon$ be a small positive parameter and let $A_{1}(t), B_{2}(t)$ be continuous matrix functions on an interval $R$. Suppose that the linear systems

$$
y^{\prime}=A_{1}(t) y \quad \epsilon z^{\prime}=B_{2}(t) z
$$

have, respectively, fundamental matrices $Y(t), Z(t)$ satisfying the inequalities

$$
\begin{aligned}
\left|Y(t) Y^{-1}(s)\right| & \leqq L \exp (\alpha|t-s|) & & \text { for } t, s \in R, \\
\left|Z(t) P Z^{-1}(s)\right| & \leqq L \exp (-\mu(t-s) / \epsilon) & & \text { for } t \geqq s, \\
\left|Z(t)(I-P) Z^{-1}(s)\right| & \leqq L \exp (-\mu(s-t) / \epsilon) & & \text { for } s \geqq t,
\end{aligned}
$$

where $L, \alpha, \mu$ are positive constants and $P$ is a projection matrix. Let $A_{2}(t), B_{1}(t)$ be any bounded continuous matrix functions on $R$. Then there exists $\epsilon_{0}>0$ such that for $0<\epsilon \leqq \epsilon_{0}$ there exists a continuously differentiable matrix function $T(t)$ which satisfies the equation

$$
T^{\prime}=\epsilon^{-1} B_{2}(t) T-T A_{1}(t)-T A_{2}(t) T+\epsilon^{-1} B_{1}(t)
$$

and which is bounded on $R$. Moreover the change of variable $z=u+T(t) y$ transforms the system

$$
y^{\prime}=A_{1}(t) y+A_{2}(t) z, \quad \epsilon z^{\prime}=B_{1}(t) y+B_{2}(t) z,
$$

into the (simpler) system

$$
\begin{aligned}
y^{\prime} & =\left[A_{1}(t)+A_{2}(t) T(t)\right] y+A_{2}(t) u, \\
\epsilon u^{\prime} & =\left[B_{2}(t)-\epsilon T(t) A_{2}(t)\right] u .
\end{aligned}
$$

Proof. Consider the integral equation $T(t)=S T(t)$ where

Received by the editors January 27, 1969.

1 This acknowledges in part the support of the N. R. C. Canada under the grant No. 5593. 


$$
\begin{aligned}
S T(t)= & \int_{a}^{t} Z(t) P Z^{-1}(s)\left[-T(s) A_{2}(s) T(s)+\epsilon^{-1} B_{1}(s)\right] Y(s) Y^{-1}(t) d s \\
& -\int_{t}^{b} Z(t)(I-P) Z^{-1}(s)\left[-T(s) A_{2}(s) T(s)\right. \\
& \left.\quad+\epsilon^{-1} B_{1}(s)\right] Y(s) Y^{-1}(t) d s,
\end{aligned}
$$

where $a$ and $b$ are respectively the left and right end points of the interval $R$.

Using (1), (2) and the identity

$$
T A_{2} T-\widetilde{T} A_{2} \widetilde{T}=(T-\tilde{T}) A_{2} T+\widetilde{T} A_{2}(T-\tilde{T})
$$

we obtain

$$
\begin{aligned}
& |S T(t)-S \widetilde{T}(t)| \leqq L^{2}\left[\int_{a}^{t} \exp \left(-\left(\mu \epsilon^{-1}-\alpha\right)(t-s)\right) d s\right. \\
& \left.\quad+\int_{t}^{b} \exp \left(-\left(\mu \epsilon^{-1}-\alpha\right)(s-t)\right) d s\right] \cdot\|T-\widetilde{T}\|\left\|A_{2}\right\|(\|T\|+\|\widetilde{T}\|)
\end{aligned}
$$

and hence

$$
\|S T(t)-S \tilde{T}(t)\| \leqq 2 L^{2} \epsilon(\mu-\epsilon \alpha)^{-1}\left\|A_{2}\right\|(\|T\|+\|\tilde{T}\|)\|T-\tilde{T}\| .
$$

Choose $\epsilon_{0}>0$ so small that

$$
\epsilon_{0} \alpha \leqq \mu / 2, \quad \epsilon_{0}\left(8 L^{2} \mu^{-1}\right)^{2}\left\|A_{2}\right\|\left\|B_{1}\right\| \leqq 1 / 2 .
$$

If $\|T\|,\|\widetilde{T}\| \leqq \rho$ where $\rho=8 L^{2} \mu^{-1}\left\|B_{1}\right\|$, it follows that for $0<\epsilon \leqq \epsilon_{0}$

$$
\|S T(t)-S \widetilde{T}(t)\| \leqq 8 L^{2} \mu^{-1} \epsilon \rho\left\|A_{2}\right\|\|T-\tilde{T}\| \leqq\|T-\widetilde{T}\| / 2 .
$$

Similarly we obtain

$$
\begin{aligned}
\|S T(t)\| & \leqq 2 L^{2}(\mu-\epsilon \alpha)^{-1}\left[\epsilon\left\|A_{2}\right\| \rho^{2}+\left\|B_{1}\right\|\right] \\
& \leqq 4 L^{2} \mu^{-1} \epsilon\left\|A_{2}\right\| \rho^{2}+\rho / 2 \leqq \rho / 4+\rho / 2<\rho .
\end{aligned}
$$

It follows by the contraction principle that the integral equation $T(t)=S T(t)$ has a unique solution $T(t)$ in $\|T\| \leqq \rho$. It is easily seen by differentiation that $T(t)$ is indeed a solution of (3). Consequently the change of variable $z=u+T(t) y$ transforms system (4) into system (5) and the theorem is proved.

In many papers $[1],[2],[3],[4],[6],[7],[8],[9]$ on singular perturbations involving the system

$$
y^{\prime}=f(t, \epsilon, y, z), \quad \epsilon z^{\prime}=g(t, \epsilon, y, z),
$$

the following hypothesis is encountered: 
(A) The matrix $U(t)=g_{z}^{-1}(t) g_{y}(t)$ has a continuous (and bounded) derivative on an $t$-interval $R$. Here $g_{y}(t)$ denotes the Jacobian matrix $g_{y}[t, 0, \bar{y}(t), \bar{z}(t)]$ where $\bar{y}(t), \bar{z}(t)$ is the solution of the degenerate system obtained by formally setting $\epsilon=0$ in (6).

The hypothesis $(A)$ is required to enable one to make the change of variables

$$
v=y-\bar{y}(t), \quad w=z-\bar{z}(t)+U(t) v,
$$

in order to transform the system (6) into the system

$$
\begin{aligned}
v^{\prime} & =A_{1}(t) v+A_{2}(t) w+F(t, \epsilon, v, w), \\
\epsilon w^{\prime} & =B_{2}(t) w+G(t, \epsilon, v, w),
\end{aligned}
$$

where

$$
\begin{aligned}
& A_{1}(t)=f_{y}(t)-f_{z}(t) U(t), \\
& A_{2}(t)=f_{z}(t), \\
& B_{2}(t)=g_{z}(t),
\end{aligned}
$$

and

$$
F=f(t, \epsilon, \bar{y}+v, \bar{z}+w-U v)-f(t, 0, \bar{y}, \bar{z})-A_{1}(t) v-A_{2}(t) w
$$

and $G$ is defined analogously. The functions $F, G$ are continuous in $(t, \epsilon, v, w)$ and such that

$$
|F(t, \epsilon, 0,0)|+|G(t, \epsilon, 0,0)| \rightarrow 0 \text { as } \epsilon \rightarrow 0 .
$$

Moreover to any $\delta>0$ there is a corresponding $\gamma(\delta)>0$ such that

$$
\begin{aligned}
& |F(t, \epsilon, v, w)-F(t, \epsilon, \tilde{v}, \tilde{w})| \leqq \delta(|v-\tilde{v}|+|w-\tilde{w}|), \\
& |G(t, \epsilon, v, w)-G(t, \epsilon, v, w)| \leqq \delta(|v-\tilde{v}|+|w-\tilde{w}|),
\end{aligned}
$$

for $t \in R$, if $|v|,|\tilde{v}|,|w|,|\tilde{w}|, \epsilon \leqq \gamma(\delta)$,

If (7) is used without the term $U(t) v$ then the system (6) is reduced to

$$
\begin{aligned}
v^{\prime} & =A_{1}(t) v+A_{2}(t) w+F_{1}(t, \epsilon, v, w), \\
\epsilon w^{\prime} & =B_{1}(t) v+B_{2}(t) w+G_{1}(t, \epsilon, v, w),
\end{aligned}
$$

where $B_{1}(t)=g_{\nu}(t)$. This system is less tractable than the system (8) owing to the presence of the term $B_{1}(t) v$. The effect of the term $U(t) v$ in (7) is to remove $B_{1}(t) v$ from (9).

The following hypotheses are also assumed or implied in the papers cited above: The functions $f, g$ and their partial derivatives with 
respect to $y, z$ are uniformly continuous and bounded on $R$; also the linear system

$$
y^{\prime}=A_{1}(t) y
$$

has a fundamental matrix $Y(t)$ satisfying the inequality (1); finally, there exists $\mu>0$ such that every eigenvalue of $g_{z}(t)$ has absolute value $\geqq 2 \mu$ for $t \in R$. It follows from Lemma 1 [4] that for sufficiently small $\epsilon>0$ the linear system

$$
\epsilon z^{\prime}=B_{2}(t) z
$$

has a fundamental matrix $Z(t)=Z(t, \epsilon)$ such that

$$
\begin{aligned}
& \left|Z(t) P Z^{-1}(s)\right| \leqq L \exp (-\mu(t-s) / \epsilon) \text { for } t \geqq s, \\
& \left|Z(t)(I-P) Z^{-1}(s)\right| \leqq L \exp (-\mu(s-t) / \epsilon) \quad \text { for } s \geqq t,
\end{aligned}
$$

where $L$ is a positive constant independent of $\epsilon$ and $P$ is the projection

$$
P=\left(\begin{array}{ll}
I_{k} & 0 \\
0 & 0
\end{array}\right)
$$

$k$ being the number of eigenvalues of $g_{z}(t)$ with negative real part.

We proceed now to show that it is not necessary at all to impose hypothesis (A) to reduce system (6) into system (8). In fact, it follows from the theorem above that there exists a bounded, continuously differentiable matrix function $T(t)$ on $R$ such that

$$
T^{\prime}=\epsilon^{-1} B_{2}(t) T-T A_{1}(t)-T A_{2}(t) T+\epsilon^{-1} B_{1}(t) .
$$

The change of variable $w=u+T(t) v$ then transforms (9) into

$$
\begin{aligned}
v^{\prime} & =A_{1}(t) v+A_{2}(t) u+\tilde{F}(t, \epsilon, v, u), \\
\epsilon u^{\prime} & =B_{2}(t) u+G(t, \epsilon, v, u),
\end{aligned}
$$

where

$$
\begin{aligned}
& \tilde{F}(t, \epsilon, v, u)=F_{1}(t, \epsilon, v, w)+A_{2}(t) T(t) u \\
& \tilde{G}(t, \epsilon, v, u)=G_{1}(t, \epsilon, v, w)-\epsilon T(t) A_{2}(t) u-\epsilon T(t) F_{1}(t, \epsilon, v, w) .
\end{aligned}
$$

The system (10) is of the same form as the system (8), since $\tilde{F}$ and $\tilde{G}$ satisfy conditions of the same type as $F$ and $G$ of (8).

\section{REFERENCES}

1. V. F. Butuzov, Asymptotic behaviour of solutions of differential equations with a small parameter in the derivative on a semi-infinite interal, Vestnik Moskov, Univ. Ser. I Mat. Meh. No. 1 (1965), 16-25.

2. K. W. Chang, Almost periodic solutions of singularly perturbed sy'stems of differential equations, J. Differential Equations 4 (1968), 300-307. 
3. - Two problems in singular perturbations of differential equations, J. Austral. Math. Soc. (to appear).

4. K. W. Chang and W. A. Coppel, Singular perturbations of initial value problems over a finite interval, Arch. Rational Mech. Anal. 32 (1969), 268-280.

5. W. A. Coppel, Dichotomies and reducibility, J. Differential Equations 3 (1967), $500-521$.

6. L. Flatto and N. Levinson, Periodic solutions of singularly perturbed systems, J. Rational Mech. Anal. 4 (1955), 943-950.

7. J. K. Hale and G. Seifert, Bounded and almost periodic solutions of singularly perturbed equations, J. Math. Anal. Appl. 3 (1961), 18-24.

8. J. J. Levin, Singular perturbations of nonlinear systems of differential equations related to conditional stability, Duke Math. J. 23 (1956), 609-620.

9. - The asymptotic behaviour of the stable initial manifolds of a system of nonlinear differential equations, Trans. Amer. Math. Soc. 85 (1957), 357-368.

10. L. Markus, Continuous matrices and the stability of differential systems, Math. Z. 62 (1955), 310-319.

\section{UNIVERSITY OF CALGaRy}

